
económico e internacionalización de empresas: una revisión de literatura. Económicas CUC, 38(1), 185-206. DOI: http:// dx.doi.org/10.17981/econcuc.38.1.09

\title{
Institucionalismo económico e internacionalización de empresas: una revisión de literatura ${ }^{1}$
}

\section{Institutional economics and internationalization of companies: a literature review}

DOI: 10.17981/econcuc.38.1.09

\author{
David Andrés Camargo Mayorga ${ }^{2}$ \\ Octavio Cardona García ${ }^{3}$ \\ Gustavo Enrique Mira Alvarado ${ }^{4}$
}

\begin{abstract}
Resumen
Este artículo presenta una revisión literaria sobre los procesos institucionales subyacentes al proceso de internacionalización empresarial, para ello se efectúa una recopilación de 57 documentos escritos entre los años 1937 y 2015 . La clasificación de la literatura se realizó teniendo como punto de partida las categorías de internacionalización definidas en Arbeláez y Serna (2002). De las posturas teóricas al respecto, se pueden destacar la evolución interna de cada compañía durante el proceso de internacionalización, la necesidad de cooperar para disminuir la incertidumbre, el efecto de la cultura y la aparición de instituciones principalmente informales.
\end{abstract}

Palabras clave: Internacionalización de empresas; Institucionalismo; Categorías de internacionalización.

Recibido: 11/4/2017 Devuelto para revisión: 19/5/17 Aceptado: 5/6/2017

\footnotetext{
${ }^{1}$ Este artículo es un producto derivado del proyecto de investigación "Internacionalización de empresas de servicios: un estudio desde el enfoque de la teoría institucional" INV-ECO- 2076, financiado por la Vicerrectoría de Investigaciones de la Universidad Militar Nueva Granada en la vigencia 2016.

${ }^{2}$ Economista y Magister en Educación. Docente de tiempo completo de la Facultad de Ciencias Económicas. Miembro del Grupo de Estudios Contemporáneos en Contabilidad Gestión y Organizaciones-Universidad Militar Nueva Granada. Correo electrónico: david.camargo@unimilitar.edu.co

${ }^{3}$ Economista y joven investigador adscrito al proyecto de investigación INV-EC0-2076. Correo electrónico: octavio.cardona.g@gmail.com

${ }^{4}$ Contador público y especialista en educación de la Universidad Militar Nueva Granada. Magíster en derecho con énfasis en tributación de la Universidad Externado de Colombia. Miembro del grupo GECS. Correo electrónico: gustavo.mira@unimilitar.edu.co
} 


\begin{abstract}
This article sets forth a literature review about the underlying institutional processes present in internationalization processes of companies. To do so, 57 documents written between 1937 and 2015 were collected. The classification of the literature was conducted using the categories of internationalization defined in Arbelaez \& Serna (2002) as a starting point. About the theoretical postures, aspects regarding the internal evolution of each company during the process of internationalization, the need of cooperation in order to reduce uncertainty, the effect of culture and the emergence of the mainly informal institutions can be highlighted.
\end{abstract}

Keywords: Firm internationalization; Institutionalism; Internationalization categories.

\title{
Introducción
}

Las instituciones entendidas como las limitaciones ideadas por las personas para dar forma a sus interacciones (North, 1993) se relacionan con los procesos de internacionalización porque van apareciendo conforme la firma va desarrollando procesos que le permiten abarcar nuevos mercados.

Una característica fundamental de las instituciones es que permiten reducir la incertidumbre. Esto precisamente es lo que buscan las empresas cuando desarrollan procesos institucionales que les permiten ejecutar labores en otros países, convirtiéndose en generadoras de desarrollo de capital humano e inclusión social con impacto en las diferentes sociedades por su capacidad de adaptación en diversos ámbitos mundiales. (Pacheco, 2013) 
Por otro lado, la internacionalización puede entenderse como un conjunto de operaciones económicas que son desarrolladas por alguna empresa por fuera de su territorio o país. Según Londoño (2015), existen tres posibles objetivos por los cuales las empresas desarrollan un proceso de internacionalización, el primero es incrementar sus ventas; el segundo es adquirir más recursos; y en un tercer lugar, minimizar riesgos.

En suma, Larrinaga (2005), Botero, Álvarez Pareja y González (2012), Verdú, Puig y Gómez (2008) y Londoño (2015) atribuyen la necesidad de internacionalización que tienen las empresas al contexto actual de globalización. Un mundo globalizado representa nuevas oportunidades, pero también involucra retos para toda la industria comercial en el mundo (Cifuentes, 2013).

Por su parte, Larrinaga (2005) conceptualiza la internacionalización como una estrategia corporativa cuyo objetivo es la diversificación geográfica a nivel internacional. Sin embargo, dicho proceso es evolutivo, gradual y dinámico que con el tiempo cambia las diferentes cadenas de valor de la empresa, y por supuesto, su estructura interna.

En el mismo sentido, Johanson y Wiedersheim (1975) la definen como un proceso donde las firmas se desarrollan en un mercado doméstico, pero van asumiendo decisiones incrementales que tienen como consecuencia la presencia de las mismas a nivel internacional.

Un cambio importante en la forma cómo se entiende la internacionalización radica en que la firma dejó de estudiarse como un agente individual y aislado que tenía transformaciones internas para ahora ser analizada como un sujeto que desarrolla formulas institucionales con proyección internacional que permiten tener una visión más ágil y flexible de las capacidades competitivas en un contexto de constante cambio (Davidson y McFetridge, 1985).

Dado que varios autores lo definen como un suceso evolutivo, este proceso puede ser considerado desde la institucionalidad implícita en el mismo, es decir que, durante el periodo de internacionalización, las empresas generan relaciones institucionales que les permiten llevar sus servicios a mercados externos. Por ejemplo, en el documento realizado por di Filippo (2008), se menciona cómo el comercio internacional ha obedecido a reglas de juego 
definidas por organizaciones supranacionales como la Organización Mundial del Comercio (OMC). Las reglas allí definidas son entendidas como instituciones formales y son un marco referencial a la hora de establecer estrategias comerciales con el extranjero.

Adicionalmente, Root (1994) precisa las estrategias de entrada a comercios internacionales, como pactos de carácter institucional, que facilitan el ingreso de diferentes bienes, servicios, recursos físicos, tecnológicos, de personal, directivos, financieros, entre otros, a países que fueron estratégicamente seleccionados por cada compañía.

Sin embargo, este tipo de instituciones no son necesariamente formales en todos los casos, de hecho, como se verá más adelante, algunas empresas buscan acceder primero a mercados con comportamientos psicológicos y culturales similares. Esto se debe a que las personas de estos mercados tienen formas de relacionarse parecidas a las del lugar de origen de la empresa.

El presente es un artículo de revisión que busca encontrar cuáles procesos institucionales subyacen al proceso de internacionalización de las empresas. El documento aborda la literatura existente para el tema de internacionalización e institucionalismo de forma análoga para luego cruzar ambas posturas en diferentes modelos teóricos usando como metodología la clasificación dentro de los modelos establecidos por Arbeláez y Serna (2002), pero también se presentará un nuevo modelo correpondiente a una categoría que, bajo el criterio de los autores de este trabajo, no fue considerado por la literatura anterior.

El hecho más destacable que surge del proceso de consulta es que varios autores consideran el proceso de internacionalización como un proceso evolutivo para la firma, de constante aprendizaje y cambio gradual que está sujeto a condiciones predefinidas en el mercado, como reglas de juego o determinismos de tipo cultural.

Lo anterior es importante porque, como se verá en los siguientes apartados, todos los modelos implican un proceso de adaptación por parte de las empresas a condiciones distintas a las de su economía doméstica. Esto significa que las compañías se enfrentan al riesgo inherente de la incertidumbre de penetrar otras economías. Con el propósito de eliminar dicha incertidumbre, aparecen las instituciones que permiten a la empresa ejercer su función internacional. 
El presente artículo se organiza de la siguiente forma: primero, una introducción al tema de estudio; segundo, se desarrollan unas consideraciones iniciales sobre la técnica empleada para recolectar la bibliografía, clasificarla y organizarla ; tercero, se presentan los modelos de internacionalización planteados por Arbeláez y Serna (2002), de los cuales emanan las categorías de análisis; cuarto, se clasifican los documentos según las categorías de análisis definidas; y quinto, se plantean unas consideraciones finales.

\section{Consideraciones iniciales}

Esta revisión de literatura comprende un periodo de observación entre 1937 y 2015, en el cual han ido evolucionando los conceptos relacionados con la internacionalización de las empresas, adquiriendo rasgos multidisciplinarios entre la administración, el marketing y, por supuesto, la economía.

Para la revisión de artículos, libros y ponencias en eventos académicos, en una primera instancia, se utilizaron combinaciones booleanas de los siguientes términos: internacionalización (internacionalization), institución (institution), proceso institucional (institutional process) y globalización (globalization).

Las búsquedas de artículos fueron realizadas en las siguientes bases de datos:

Tabla 1

Principales bases de datos académicas consultadas

\begin{tabular}{lc}
\hline \multicolumn{1}{c}{ Base } & $\begin{array}{c}\text { Número de } \\
\text { documentos }\end{array}$ \\
\hline Jstor & 15 \\
EconPapers & 10 \\
Dialnet & 5 \\
\hline
\end{tabular}

Fuente: elaboración propia

Una vez realizada la búsqueda en diferentes bases de datos académicas, se procedió a seleccionar los artículos más pertinentes y a consultar las fuentes allí citadas que tuvieran relación con el tema consultado dado que algunas de ellas son principalmente libros o do- 
cumentos electrónicos que no se encuentran alojados en la red. A partir de las fuentes más importantes, se realizó una nueva búsqueda, pero con tres criterios más: costos de transacción (transaction cost), cooperación (cooperation) y evolución (evolution).

Tabla 2

Número de artículos y documentos por temática.

\begin{tabular}{lc}
\hline \multicolumn{1}{c}{ Criterio } & $\begin{array}{c}\text { Número de } \\
\text { documentos }\end{array}$ \\
\hline Internacionalización e institucionalismo & 16 \\
Estrategias de internacionalización & 18 \\
Libros y otros documentos relacionados & 19 \\
\hline
\end{tabular}

Fuente: elaboración propia

En última instancia, se clasificó cada una de las revistas consultadas de acuerdo con su especialidad; los resultados de la clasificación se muestran en la siguiente tabla:

Tabla 3

Lista de revistas por especialidad.

\begin{tabular}{|c|c|c|}
\hline Economía & Administración y Managment & Mercadeo \\
\hline $\begin{array}{l}\text { Económica } \\
\text { Información Comercial } \\
\text { Española ICE: Revista de } \\
\text { economía } \\
\text { Oxford Bulletin of Economics } \\
\text { and Statistics } \\
\text { Quarterly Journal of Economics } \\
\text { Review of World Economics } \\
\text { Revista Asturiana de Economía } \\
\text { (RAE) } \\
\text { The Journal of Law \& } \\
\text { Economics } \\
\text { Weltwirtschaflitches }\end{array}$ & $\begin{array}{l}\text { Academy of International Business } \\
\text { AD-minister } \\
\text { Cuadernos de Gestión } \\
\text { Estudios Internacionales } \\
\text { Harvard Business Review } \\
\text { Journal of International Business } \\
\text { Studies } \\
\text { Journal of Management Studies } \\
\text { Journal of Product Innovation } \\
\text { Management } \\
\text { Management Science } \\
\text { MIT Sloan Management Review } \\
\text { Strategic Management Journal } \\
\text { Universia Business Review }\end{array}$ & $\begin{array}{l}\text { International Marketing } \\
\text { Review } \\
\text { Journal of Marketing } \\
\text { Research } \\
\text { Journal of the Academy of } \\
\text { Marketing Science }\end{array}$ \\
\hline
\end{tabular}

Fuente: elaboración propia 


\section{Modelos de internacionalización}

Para incluir dentro de un mismo marco conceptual el proceso institucional y el de internacionalización, a continuación, se presentan los modelos de internacionalización elaborados por Arbeláez y Serna (2002):

Modelo U (Uppsala). Es un modelo que trata de explicar la internacionalización como un proceso que se va dando gradualmente en donde las compañías primero fortalecen su presencia al interior del territorio de origen para luego acceder a mercados psicológicamente cercanos.

Luego de los mercados psicológica y geográficamente cercanos, la empresa puede acceder a desarrollar operaciones por fuera de estos ámbitos contiguos. Cuando la empresa se encuentra en esta última etapa, debe trabajar principalmente en dos aspectos: el desarrollo del mercado y la diversificación.

Modelo B. Concibe la internacionalización desde una perspectiva de costo-beneficio. Tiene en cuenta que cualquier proceso de comercio exterior genera costos de transacción que al mismo tiempo derivan en externalidades negativas que deben ser solucionadas mediante el Teorema de Coase o la aplicación de impuestos pigouvianos.

Aunque todo esto implica necesariamente la negociación entre los diferentes empresarios, para la aplicación de los impuestos es necesaria la intervención del gobierno en forma de subsidios o salvaguardias.

Modelo H. En el modelo H, la internacionalización se fundamenta en la competitividad de la industria, es decir que antes de llevar a cabo una labor internacional, la firma debe resolver los problemas competitivos locales.

En este modelo son cuatro los factores que determinan la competitividad de una empresa en un contexto internacional: condiciones de los factores, condiciones de la demanda, naturaleza de la industria y la estrategia empresarial.

Modelo W. El modelo W es esencialmente un modelo de "coopetencia". Las empresas elaboran estrategias de cooperación que les facilita el ingreso a nuevos mercados, incluso cuando entren a competir al interior del territorio. 
Este modelo se caracteriza por la constante búsqueda que tienen las empresas para hallar una "sinergia" que les permita desenvolverse en el nuevo mercado, que, dependiendo sus condiciones, ejercerá una influencia sobre las firmas para que asuman una posición cooperativa o competitiva.

Modelo $\mathbf{R}^{2}$. Es un modelo que pretende combinar el proceso de internacionalización con la ecléctica. La primera parte describe la creación y fortalecimiento de los vínculos entre las firmas, cuyo resultado es una proyección progresiva hacia los mercados extranjeros. La segunda, se encarga de explicar las causas de la internacionalización y las estrategias de localización en los mercados internacionales.

Modelo M. En el modelo M, las operaciones de comercio internacional ocurren dentro de un marco cultural definido que se traduce en aspectos políticos, sociales, económicos, tecnológicos, lingüísticos, religiosos y éticos.

En este modelo, la cultura no es simplemente una variable, sino que es el medio sobre el que se desenvuelven los procesos de internacionalización. Este aspecto es coincidente en varios de los modelos, pues las culturas psicológicamente cercanas representan un riesgo mucho menor para el empresario.

Modelo E. Este es un modelo que destaca la capacidad emprendedora e innovadora en los procesos que se relacionan con la internacionalización. Esta capacidad emprendedora e innovadora se destaca en las nuevas formas de hacer negocios para lograr mercados externos.

Dentro de esta categoría se encuentran grandes empresas de tecnología que fundamentan su negocio en el comercio electrónico y que utilizan la plataforma web para acercarse a nuevos clientes, adquirir nuevas empresas o hacer algún tipo de alianza estratégica.

\section{Clasificación por categoría de análisis}

Modelo U (Uppsala). El modelo U atribuye su nombre a las investigaciones realizadas principalmente por Jan Johanson y Finn Wierdersheim, de la universidad de Uppsala, quienes describieron el proceso de internacionalización que se podía observar a mediados de los años setenta en diferentes firmas suecas. 
El mencionado modelo fue probablemente el primero en explicar la internacionalización de la empresa a partir del modo de operación interna. Este proceso se puede exponer con la matriz que fue propuesta por Ansoff (1957), principalmente con las celdas relativas al desarrollo del mercado y a la diversificación. Hay que tener en cuenta que este modelo es normalmente aplicado por empresas que llegaron a ser multinacionales (Pla y León, 2004).

La explicación central del modelo es que la expansión internacional se da como un proceso secuencial (Bilkey y Tesar, 1977; Leonidou y Katsikeas, 1996) en el que las empresas gradualmente van adquiriendo experiencia hasta establecerse totalmente en otro país distinto al de origen (Verdú, Puig y Gómez, 2008). No obstante, para que las empresas puedan tener presencia internacional, primero debe haber un fortalecimiento a nivel interno, luego de esto, tratan de acceder a mercados "psicológicamente cercanos" (Londoño, 2015).

Análogamente, Johanson y Vahlne (1990) afirman que la internacionalización de la firma se va dando gradualmente, como un proceso que se podría considerar evolutivo. De hecho, como aparece en el documento de Arbeláez y Serna (2002), el modelo está profundamente desarrollado bajo la consideración de que las firmas toman decisiones en condiciones de racionalidad acotada cuando se enfrentan a la disyuntiva entre crecimiento y riesgo.

En el mismo orden de ideas, North (1993) asevera que la función principal de las instituciones es disminuir la incertidumbre. Esto es un factor fundamental para argumentar que el modelo U describe esencialmente un proceso de construcción institucional, que en este caso se da a nivel interno en una organización: la firma.

Ahora bien, el lector debe tener en cuenta que en el periodo de estudio en el que fue desarrollándose este modelo de internacionalización no se contaba con los avances tecnológicos con los que se cuenta actualmente, algo que representaba un obstáculo para la mayoría de las empresas de la época. No obstante, a partir de los cambios que ha habido, actualmente se han desarrollado una variedad de modelos que se ajustan mejor a otras propuestas de valor que han ido apareciendo desde los años ochenta. Algunos de esos modelos se explican a continuación.

Modelo B. El modelo B es una explicación desde el punto de vista de la economía clásica. Fundamentalmente, interpreta el proceso de internacionalización como una relación costobeneficio, pero enmarca su análisis dentro de la teoría de costes de transacción. 
El análisis del modelo se centra en los costos inherentes que se generan a la hora de firmar contratos entre dos agentes de diferentes países cuando se busca internacionalizar cualquier negocio. Dichos acuerdos, pueden generar diferentes tipos de externalidades que deben ser resueltas, ya sea por el Teorema de Coase o mediante impuestos Pigouvianos.

Aunque este modelo tiene un marco referencial bastante robusto ${ }^{1}$, su ejecución operacional resulta muy difícil de aplicar dado que no se pueden conocer a priori los costos transaccionales en los que se incurre hasta que no se complete una primera operación internacional (Dunning, 1993).

Este modelo se enmarca perfectamente dentro la definición de institución formal que se define en el trabajo de Williamson (1979), pues da un valor superlativo a los contratos que se ejecutan entre los agentes y a los efectos directos e indirectos que generan.

Modelo H. El modelo H se encuadra dentro de la misma línea económica que el anterior, pero, en este caso, la internacionalización se fundamenta en la competitividad industrial. Obtiene su valor teórico especialmente desde el "diamante de ventaja nacional" planteado por Porter (1990).

De esta manera, cuatro elementos son los que determinan la competitividad de la empresa en un entorno internacional, como ya se mencionó: primero, las condiciones de los factores; segundo, las condiciones de la demanda; tercero, la naturaleza de las industrias relacionadas y cuarto, la estrategia de la empresa.

Aunque es un modelo bastante útil para acercarse al proceso de internacionalización, han aparecido críticas, como las de Van Den Bulke (1995) y Jegers (1995), quienes consideran excesivo el uso del diamante de Porter como explicación de la base de la empresa multinacional y se cuestionan sobre la pertinencia de los diamantes para toda clase de paises en un periodo de tiempo ilimitado. Al respecto, han aparecido propuestas, que ponen en consideración una teoría de "doble diamante", es decir, que considere el diamante de la economía doméstica de la empresa y el diamante extranjero (Chang Moon, Rugman y Verbeke, 1995).

\footnotetext{
1 Pueden consultarse los trabajos de Anderson y Gatignon (1986); Reid (1983); Klein (1989); Hill y Kim (1988); y Klein, Frazier y Roth (1990).
} 
El marco institucional de este modelo no es tan claro, sin embargo, puede dilucidarse algo del mismo en los dos últimos elementos: la naturaleza de la industria y la estrategia de la empresa. En esto último, toman bastante importancia las llamadas "cinco fuerzas de Porter", que se resumen en el poder de negociación de los clientes; la rivalidad entre las empresas; la amenaza de los nuevos entrantes; el poder de negociación de los proveedores y la amenaza de los productos sustitutos. Pero dichos procesos se explican mejor en modelos como el B (para el caso de la industria) y el U (para el caso de la firma en particular).

Modelo W. Este modelo fundamenta el proceso de internacionalización en una serie de estrategias empresariales que incluyen la cooperación entre las firmas, esto con el objetivo de expandir su mercado (Londoño, 2015). Aquí el proceso de internacionalización se presenta como resultado de la racionalización y estructuración de la competencia y la cooperación con el propósito de balancearlas para encontrar una sinergia empresarial que facilite el acceso a los mercados foráneos (Arbeláez y Serna, 2002).

Estos aspectos son muy similares al neologismo "coopetencia", que aparece por primera vez en la obra de Nalebuff, Brandenburger y Brandenburger (1997). En su trabajo, los autores explican por qué el mercado funciona como un juego de estrategia en el que es necesario competir y cooperar con los demás agentes, dependiendo de las circunstancias.

De igual forma, Rialp, Martínez y Rialp (2005) destacan que los nuevos métodos de cooperación han puesto al alcance de cualquier empresa la oportunidad de exportar. Esto significa que la adecuada elección de socios, el ámbito de los acuerdos y la forma de contratación se han hecho cada vez más importantes para las firmas que quieren tener una presencia comercial en el extranjero (Alonso, 1993).

El modelo W, en últimas, es un modelo de "coopetencia”, pues describe dicho proceso, sólo que aplicado al mercado internacional. Dependiendo la coyuntura del mercado, los agentes asumirán una posición cooperativa o competitiva, con el fin de mantenerse y expandirse en el mercado.

Modelo $\boldsymbol{R}^{2}$. Aquí el proceso de internacionalización es explicado básicamente desde las teorías de la internacionalización y la ecléctica. Londoño (2015) explica que en una primera instancia el modelo describe la creación y fortalecimiento de vínculos empresariales para luego manifestar las causas de la internacionalización y el proceso de localización estratégica en los mercados extranjeros. 
Resulta oportuno mencionar que el paso de las teorías de internacionalización a un componente ecléctico fue un sumario incremental iniciado, primero, desde la teoría de los costes de transacción, tanto en su versión original definida por Coase (1937), en donde se describe que mientras hallan costes de transacción, las firmas tendrán una razón para existir, como en su versión ampliada, desarrollada por Williamson (1975) y Williamson (1985), en donde no sólo se describen los costos de las firmas, sino el comportamiento de las mismas con respecto a un entorno de racionalidad limitada, oportunismo, especificidad de los activos y el proceso de contrato que implicaría la combinación de dichos factores.

El segundo paso, desde la teoría de los costos de transacción, fue el de la economía de organización industrial. Aquí destacan los trabajos de Caves (1971), que trata del efecto de la inversión extranjera directa sobre el proceso industrial, y el de Porter (1990), con su aporte de la ventaja competitiva.

En tercer lugar, se llegó al institucionalismo económico. Aquí incide el trabajo pionero de Vernon (1966), pues se aleja de la explicación clásica del costo comparativo para enfocarse en la influencia que variables como la innovación, los efectos de las economías a escala, la ubicación y el ciclo de vida de los productos, el papel de la ignorancia y la incertidumbre tienen sobre los patrones de comercio.

Luego, el marco teórico fue inclinándose hacía una explicación definitiva del porqué de la actividad multinacional ${ }^{2}$. En primera instancia apareció la teoría de internalización económica, cuya idea principal es que la empresa internaliza aquellos factores del mercado que se presentan de forma ineficiente (Rugman, 1980).

Si bien es cierto que puede ser una jugada administrativa favorable, la empresa depende de su capacidad para mantener los costos internalizados por debajo de los costos de transacción que se dan en el mercado (Hennart, 1986). En otros términos, esta teoría está fundamentada en dos axiomas fundamentales: (1) las empresas eligen la ubicación que menos costos represente y (2) las empresas crecen mediante la internalización del mercado, hasta el punto en que los beneficios de una mayor internalización se compensan por los costos (Buckley, 1988).

\footnotetext{
${ }^{2}$ Se encuentran aplicaciones de este modelo en los trabajos de Buckley y Casson $(1976,1985)$; Casson (1979, 1982, 1987) y Rugman (1981, 1982).
} 
En el último escalón, se encuentra el paradigma ecléctico (Dunning, 1979, 1980, 1988). En este enfoque, Alonso (1993) asegura que hay tres factores necesarios para que la empresa se convierta en internacional: (1) la empresa debe tener alguna ventaja, usualmente en sus activos intangibles, sobre los competidores locales; (2) asumiendo que existe la anterior condición, la empresa debe decidir si aprovecha los nuevos mercados y optan por exportar, invertir o ceder, mediante licencias y contratos, sus activos diferenciales; y (3) dadas las condiciones anteriores, si la empresa decide invertir, debe asegurarse de que su inversión sea rentable de acuerdo con la localización, en caso contrario, el camino a seguir debería ser la exportación.

Por último, se hace indispensable mencionar que el enfoque ecléctico no se considera una teoría integral por ciertas limitaciones en sus argumentos, principalmente en lo que tiene que ver con la diferenciación entre las ventajas específicas de la firma y aquellas que se dan por un proceso dinámico de internalización, pues las primeras ventajas son necesariamente producto del proceso de internalización. De esta forma, el enfoque ha sido considerado "paradigma" o simplemente una disposición de factores que promueven la inversión extranjera (Itaki, 1991).

Modelo M. Según Arbeláez y Serna (2002), la internacionalización ocurre dentro de un contexto cultural definido que se materializa en aspectos políticos, tecnológicos, sociales, económicos, religiosos, éticos, lingüísticos, entre otros. Pero la cultura no sólo debe ser vista solo como el marco en donde se desenvuelven dichas actividades, sino también como un medio y un vehículo que conduce al proceso de internacionalización. Un ejemplo claro es la capacidad emprendedora entre los diferentes países, pues cada ubicación geográfica presenta dinámicas distintas; también lo es la decisión de invertir, ir en joint venture o establecerse en determinado país. Un estudio empírico al respecto se encuentra en Kogut y Singh (1988), en donde se estudian 228 entradas de capital a Estados Unidos. En el modelo probabilístico usado por los autores se encontró que cuando existe una distancia cultural mayor, los inversionistas tienen más probabilidades de elegir joint ventures que adquisiciones y compras totales.

Los aspectos que se mencionaron con anterioridad usualmente obedecen a procesos institucionales de diferente índole. Por un lado, se encuentran procesos constructivistas (como el caso de la política, las leyes, algunos aspectos económicos y sociales, la religión, la ética, etc.), y por el otro, procesos de orden espontáneo (como los aspectos culturales, lingüísticos, algunos tecnológicos, económicos y sociales). 
Estos procesos, independiente de su índole, definen las reglas de juego bajo las que los agentes asumen decisiones. Dichas reglas usualmente generan el llamado "efecto marco", que es la "aceptación pasiva de una formulación dada" por parte de un individuo (Kahneman, 2003, p. 198). Lo que significa que los sujetos asumen decisiones intuitivamente basados en la información disponible. Esa información usualmente es limitada por las instituciones, por lo tanto, el rol del marco institucional y su adecuada fiabilidad moldeará el proceso de elección individual.

Modelo E. Más allá de un modelo, lo que Arbeláez y Serna (2002) rescatan es la capacidad innovadora y emprendedora, como pilares del proceso de internacionalización. Para los autores, el modelo E de internacionalización tiene en cuenta la relación inversa entre diferenciación e integración (Lawrence y Lorsch, 1967), además viene sustentado por los pilares de los anteriores modelos.

El marco conceptual del modelo E lo ubica dentro de un enfoque ecléctico, que tiene en consideración el valor, los costos de transacción, la internacionalización, las alianzas estratégicas, la estrategia competitiva, la racionalidad limitada y la cultura, todo enmarcado en una visión de una economía basada en recursos.

Para este modelo, se pierde en primera instancia el significado de las empresas multinacionales, pues no se limita solamente a la descripción de una empresa que se encuentra en varios países (Buckley y Casson, 2003). Estos procesos son bien ejemplificados en Albaum y Duerr (2011) en los estudios de caso del primer capítulo, relacionados con empresas tecnológicas o basadas en la web (específicamente eBay y Dell).

Modelo T. El modelo $\mathrm{T}$ es un modelo de negocio que tiene en cuenta, principalmente, la tecnología basada en la plataforma web. Su característica principal es que las empresas que nacen bajo este modelo prácticamente nacen siendo internacionales. Un ejemplo simple de la anterior afirmación son las empresas (a veces unipersonales) que crean aplicaciones móviles y las suben a las tiendas de aplicaciones.

El modelo consiste en integrar una comunidad de usuarios entorno a una plataforma, sin embargo, dichos usuarios no son sólo consumidores, también se encuentran usuarios expertos o lead users (Miguel-Dávila, López y Pablos_Heredero, 2012); este tipo de usuarios, 
de acuerdo con Von Hippel (1986), se percatan de necesidades no satisfechas que aún no son percibidas por los demás consumidores, por lo tanto, tratan de solucionarlas creando un beneficio para toda la comunidad.

Estos usuarios son el factor diferencial de este modelo, autores como Franke, von Hippel, y Schreier (2006) afirman que (1) la participación de los lead users se correlaciona directamente con innovaciones de tipo comercial; (2) siempre que un usuario experto lanza al mercado algún aplicativo espera una retribución, aunque esta no sea necesariamente material y (3) la participación activa de estas personas expertas influye de forma positiva sobre la innovación del sector.

El modelo T fundamenta su ventaja en ser un modelo abierto, es decir, cualquier persona en cualquier parte del mundo puede participar en el desarrollo del producto. Para MiguelDávila, López y Pablos-Heredero (2012), este no es simplemente un proceso de externalización (outsourcing) en el que los propósitos del acuerdo se definen por adelantado.

Como resultado de lo anterior, Chesbrough (2007) explica que las redes de valor que se van generando son más fluidas que los acuerdos comerciales entre empresas, principalmente porque tienen una cantidad nada despreciable de agentes que se relacionan, interactúan e intercambian ideas y modelos de negocio.

\section{Consideraciones finales}

Luego de revisar las formas de internacionalización que se definieron en esta revisión y compararlas con los procesos institucionales, hay que destacar que corresponden principalmente a instituciones informales.

En ninguno de los modelos hay una mención explícita del gobierno o de las leyes como actores que pueden influir sobre la decisión de hacer internacionalización, por el contrario, los acuerdos empresariales, los cambios al interior de cada empresa y algunos factores relacionados con la cultura de cada nación priman sobre la decisión de ejercer o no una actividad empresarial por fuera del país de origen. 
Además, en cada uno de los modelos hay una importancia superlativa en asuntos como el cambio gradual al interior de las empresas, la necesidad de recurrir primero a mercados "psicológicamente" cercanos, la "coopetencia" y la disyuntiva entre integrarse o diferenciarse durante el proceso.

A pesar de que cada modelo tiene consideraciones sobre la firma como un agente individual, la necesidad de disminuir la incertidumbre obliga a las empresas a realizar alianzas mucho antes de entrar a cualquier economía externa, no obstante, dichas alianzas pueden generar externalidades en el país al que ingresa pues las externalidades no son un problema menor dado que no pueden estimarse antes de iniciar operaciones.

Por ello, se hace perentorio que cada una de las organizaciones que pretenda hacer internacionalización use como estrategia la capacitación de su personal para diseñar y administrar los cambios necesarios dentro y fuera de la organización, generándoles competencias y avances cognitivos que permitan a cada empleado tomar decisiones vinculadas a sus áreas respetivas (Samper y Maussa, 2014) (Kadi Montiel y Acevedo Duque, 2014), considerando, además, las herramientas gerenciales idóneas que contribuyan de manera favorable para alcanzar este objetivo (Villasmil \& Crissien, 2015).

Finalmente, para futuras investigaciones, sería pertinente realizar análisis empíricos sobre el papel que tiene el cambio tecnológico dentro de todo el proceso de internacionalización; revisar asuntos relacionados con las instituciones formales (prácticamente ausentes en los modelos revisados, con excepción de los acuerdos legales) para identificar la importancia relativa de las mismas e indagar sobre los efectos que tiene una eventual diferenciación o una integración cuando una compañía decide extender su negocio fuera de su país.

\section{Referencias}

Albaum, G. y Duerr, E. (2011). International Marketing and Export Management. Nueva Jersey: Pearson. 
Alonso, J. (1993). Internationalisation process and forms of market penetration: a dynamic proposal. Madrid: Facultad de Ciencias Económicas y Empresariales, Universidad Complutense de Madrid.

Anderson, E. y Gatignon, H. (1986). Modes of foreign entry: a transaction cost analysis and propositions. Journal of International Business Studies, 17, 1-26.

Ansoff, H. (1957). Strategies for diversification. Harvard Business Review, 35(5), 113-124.

Arbeláez, H. y Serna, H. (2002). Lessons from Four Successful Latin American Cases of Internationalization. Academy of International Business, 7.

Bilkey, W. y Tesar, G. (1977). The Export Behavior of Smaller-Sized Wisconsin Manufacturing Firms. Journal of International Business Studies, 8. 93-98. https:// doi.org/10.1057/palgrave.jibs.8490783

Botero, J., Álvarez Pareja, F. y González. (2012). Modelos de internacionalización para las pymes colombianas. AD-minister, (20), 63-90.

Buckley, P. (1988). The limits of explanation: testing the internalization theory of the multinational enterprise. Journal of International Business Studies, 19, 181-193.

Buckley, P. J. y Casson, M. (1976). The future of the multinational enterprise. London: Macmillan.

Buckley, P. J. y Casson, M. (1985). The economic theory of the multinational enterprise: selected papers. London: Macmillan.

Buckley, P. y Casson, M. (2003). The future of the multinational enterprise in retrospect and in prospect. Journal of International Business Studies, 34(2), 219-222.

Casson, M. (1979). Alternatives to the multinational enterprise. London : Macmillan.

Casson, M. (1982). The growth of international business. London: George Allen and Unwin.

Casson, M. (1987). The firm and the market. Cambridge Mass: MIT Press. 
Caves, R. (1971). International Corporations: The Industrial Economics of Foreign Investment. Economica, 38(149), 1-27.

Chang Moon, H., Rugman, A. y Verbeke, A. (1995). The generalized double diamond approach to international competitiveness. En, A. Rugman, J. Van Den Broec y A. Verbeke, Research in Global Strategic Management: Beyond the Diamond (Vol. 5). Greenwich, CT: JAI Press.

Chesbrough, H. (2007). Why companies should have open business models. MIT Sloan Management Review, 48(2), 22-28.

Cifuentes, G. (2013). Desarrollo constitucional, legal y jurisprudencia del principio de rigos subsidiario. Juridicas CUC, 9(1), 345-384.

Coase, R. (1937). The Nature of the Firm. Economica, 4(16), 386-405.

Davidson, W. y McFetridge, D. (1985). Key characteristics in the choice of international technology transfer mode. Journal of International Business Studies, 16, 5-21.

di Filippo, A. (2008). La calidad de las instituciones y su impacto sobre el comercio internacional. Estudios Internacionales, (159), 79-96.

Dunning. (1979). Explaining changing patterns of international production: in defence of the eclectic theory. Oxford Bulletin of Economics and Statistics, 275-295.

Dunning, J. (1980). Towards an Eclectic Theory of International Production: Some Empirical Tests. Journal of International Business Studies II, 9-31.

Dunning, J. (1988). The Eclectic Paradigm of International Production: A Restatement and Some Possible Extensions. Journal of International Business Studies (Spring), $1-32$.

Dunning, J. (1993). Multinational Enterprises and the Global Economy. Reading, MA: Addison-Wesley.

Franke, N., Von Hippel, E. y Schreier, M. (2006). Finding commercially attractive user innovations: A test of lead user theory. Journal of Product Innovation Management, 23(4), 301-315.

Hennart, J. (1986). What is internalization? Weltwirtschaflitches, 122(4). 791-804. 
Hill, Ch. y Kim, W. (1988). Searching for a dynamic theory of the multinational enterprise: A transaction cost model. Strategic Management Journal, 9(S1). 93104.

Itaki, M. (1991). A critical assessment of the eclectic theory of the multinational enterprise. Journal of International Business Studies, 22(3), 445-460.

Jegers, M. (1995). Are Diamonds Forever? An Industrial-Economics Perspective. En A. Rugman, J. Van Den Broec y A. Verbeke, Research in Global Strategic Management: Beyond the Diamond (Vol. 5). Greenwich, CT: JAI Press Inc.

Johanson, J. y Vahlne, J. (1990). The Mechanism of Internationalization. International Marketing Review, 7(4), 11-24.

Johanson, J. y Wiedersheim-Paul, F. (1975). The Internationalization of the Firm: Four Swedish Cases. Journal of Management Studies, 12(3), 305-322.

Kadi Montiel, O. J. y Acevedo Duque, Á. E. (2014). Liderazgo ético frente a la diversidad cultural dentro de las organizaciones con régimen disciplinario. Económicas CUC, 35(2), 75-88.

Kahneman, D. (2003). Mapas de racionalidad limitada: psicología para una economía conductual. Revista Austriana de Economía (RAE), 181-225.

Klein, S. (1989). A transaction cost explanation of vertical control in international markets. Journal of the Academy of Marketing Science, 17(3), 253-260.

Klein, S., Frazier y Roth. (1990). A transaction cost analysis model of channel integration in international markets. Journal of Marketing Research, 27, 196-208.

Kogut, B. y Singh, H. (1988). The Effect Of National Culture On The Choice Of Entry Mode. Journal of International Business Studies, 19(3), 411-432.

Larrinaga, O. (2005). La internacionalización de la empresa y la empresa multinacional: una revisión conceptual contemporánea. Cuadernos de Gestión, 5(2), 55-73. 
Lawrence, P. y Lorsch, J. (1967). Organization and Environment: Managing Differentiation and Integration. Boston: Harvard Business School Publications. https://doi.org/10.1177/002218566801000314

Leonidou, L. y Katsikeas, C. (1996). The Export Development Process: An IntegrativeReview of Empirical Models. Journal of International Business Studies, 517-551.

Londoño, C. (2015). El proceso de internacionalización de las Pymes colombianas. Un análisis a partir del planteamiento de la teoría de juegos. En, Grupo SUMAR, Aprendizajes desde la Academia sobre las Pequeñas y Medianas empresas (141165). Medellín: Fundación Universitaria María Cano.

Miguel-Dávila, M., López, P. y Pablos-Heredero, C. (2012). El sector de la telefonía móvil como modelo de negocio abierto en un contexto de innovación sistémica. Universia Business Review, (36), 48-62.

Nalebuff, B. J., Brandenburger, A. y Brandenburger, A. M. (1997). Coopetición: un revolucionario esquema mental para la gestión que combina la competencia y la cooperación, las estrategias de la teoría de los juegos que están cambiando el juego de los negocios. Madrid: Ediciones Díaz de Santos.

North, D. (1993). Instituciones, cambio institucional y desempeño económico. México, D.F.: Fondo de Cultura Económica.

Pacheco, C. (2013). Estrategias empresariales más utilizadas por las pymes en Sincelejo. Económicas CUC, 34(1), 183-202.

Pla, J. y León, F. (2004). Dirección de Empresas Internacionales. Madrid: Pearson Educación.

Porter, M. (1990). La ventaja competitiva de las naciones. Barcelona: Plaza \& Janes Editores.

Reid, S. (1983). Firm internationalization. Transaction costs and strategic choice. International Marketing Review, 43-61. 
Rialp, A., Martínez, P. y Rialp, J. (2005). El desarrollo exportador de las pymes industrialesespañolas participantes en un consorcio deexportación: un estudio de caso. Cuadernos de Gestión, 5(2), 95-116.

Root, F. (1994). Entry Strategies for International Markets. New York: Lexington Books.

Rugman, A. (1980). Internalization as a General Theory of Foreign Direct Investment: A Re-Appraisal of the Literature. Review of World Economics, 116(2), 365-379.

Rugman, A. (1981). Inside the multinationals. New York: Columbia University Press.

Rugman, A. (1982). New theories of the multinational enterprise. London: Croom Helm.

Samper, J. y Maussa, E. (2014). Desarrollo moral y competencias ciudadanas en la juventud universitaria. Jurídicas CUC, 10(1), 43-60.

Van Den Bulke, D. (1995). The Strategic Management of Multinationals in a TriadBased World Economy. En A. Rugman, J. Van Den Broec y A. Verbeke, Research in Global Strategic Management: Beyond the Diamond (Vol. 5). Greenwich, CT: JAI Press.

Verdú, F., Puig, A. y Gómez, V. (2008). Internacionalización, servicios y política de innovación: El papel de los Centros Tecnológicos. Información Comercial Española ICE: Revista de economía, (844), 155-165.

Vernon, R. (1966). International Investment and International Trade in the Product Cycle. Quarterly Journal of Economics, 190-207.

Villasmil, M. y Crissien, T. (2015). Cambio de paradigma en la gestión universitaria basado en la teoría y práxis de la reingeniería. Económicas CUC, 36(1), 127-142.

Von Hippel, E. (1986). Lead Users: A Source of Novel Product Concepts. Management Science, 32(7), 791-805. 
Williamson, O. (1975). Markets and Hierarchies. New York: The Free Press.

Williamson, O. (1979). Transaction-Cost Economics: The Governance of Contractual Relations. The Journal of Law \& Economics, 22(2), 223-261.

Williamson, O. (1985). The Economic Institutions of Capitalism. New York: The Free Press. 